Revue d'histoire de l'enfance « irrégulière »

Le Temps de l'histoire

$4 \mid 2002$

Images de l'enfance et de la jeunesse "irrégulières »

\title{
La jeunesse irrégulière dans la télévision française des années soixante : une absence troublante
}

Marie-Françoise Lévy

\section{(2) OpenEdition}

Édition électronique

URL : http://journals.openedition.org/rhei/53

DOI : $10.4000 /$ rhei.53

ISBN : 978-2-7535-1642-7

ISSN : $1777-540 \mathrm{X}$

Éditeur

Presses universitaires de Rennes

Édition imprimée

Date de publication : 15 novembre 2002

Pagination : 73-86

ISSN : 1287-2431

Référence électronique

Marie-Françoise Lévy, « La jeunesse irrégulière dans la télévision française des années soixante : une absence troublante », Revue d'histoire de l'enfance « irrégulière » [En ligne], 4 | 2002, mis en ligne le 03 mai 2007, consulté le 04 décembre 2020. URL : http://journals.openedition.org/rhei/53 ; DOI : https:// doi.org/10.4000/rhei.53 


\section{La jeunesse irrégulière dans la télévision française des années soixante : une absence troublante}

Pourquoi prendre pour sujet les formes du traitement télévisuel de la "jeunesse irrégulière" et les modes d'intervention de la télévision française sur ce même sujet, dès lors que, sur la période étudiée, les années 1958-1968, vingt émissions au plus lui sont consacrées ? Le chiffre, en effet, est étonnamment faible si l'on prend en considération le volume horaire de la programmation annuelle ( 2.500 heures en 1958 et 4.000 heures en 1968). Par ailleurs, cette production s'avère également infime rapportée à l'ample développement sur le petit écran de magazines qui prennent, dans les années soixante, la jeunesse pour sujet et public d'émissions. Face à ce premier constat, pourquoi persister et poursuivre l'étude ? Sans doute, parce que cette forme d'absence - volontaire ou non - constitue en elle-même une question.

Ces rares émissions sur la jeunesse irrégulière peuvent, en effet, prendre sens en regard des représentations de la jeunesse proposées dans ces magazines spécifiques. C'est donc une lecture en creux qu'il s'agit, en premier lieu, d'entreprendre. Ces émissions si nombreuses sur la jeunesse dessinent, en effet, implicitement, dans le silence accordé à la jeunesse irrégulière, les caractéristiques de cette dernière. Comme si les valeurs et les normes promues - le sérieux, la responsabilité notamment - comprenaient de façon suffisamment explicite leur contre-modèle, sans avoir besoin ni de le nommer ni de le montrer. Dans un deuxième temps, une autre approche s'impose. Elle consiste dans l'analyse des documents portant précisément sur la jeunesse en difficulté, considérés comme des indicateurs de la construction médiatique - mais aussi métaphorique - d'une question sociale et de ses infléchissements au cours de la décennie. Ces deux axes de réflexion, dans leurs confrontations, permettent de poser, à
MarieFrançoise Lévy ${ }^{(1)}$

(1) Chercheur à

l'Institut d'histoire du temps présent (IHTP), CNRS.

Marie-Françoise Lévy / p. 73 à 86 
(2) Les extraits et commentaires d'émissions télévisées seront signalés par des guillemets "anglais" et l'italique.

(3) Jérôme Bourdon,

Histoire de la télévision sous De Gaulle, Paris, Anthropos, 1990, p. 27.

(4) Jean-Louis Missika, Dominique Wolton, La folle du logis. La télévision française dans les sociétés démocratiques, Paris, Gallimard, 1983.

(5) Marie-Françoise Lévy, [dir.], La télévision dans la République. Les années cinquante, éd. Complexe, Bruxelles, 1999.

(6) Hervé Michel, Les grandes dates de la télévision française, Presses universitaires de France, Paris, 1995.

(7) Si c'était vous. "Délinquance juvénile", scénario de Marcel titre prospectif et comme pierres d'attente, quelques observations et remarques..$^{(2)}$

\section{La jeunesse : une permanence du sujet dans les programmes de télévision}

Par l'ordonnance du 4 février 1959, la Radio télévision française devient un établissement public à caractère industriel et commercial, " géré, sous l'autorité du ministère de l'Information, par un directeur général ${ }^{(3)} \mathrm{La}$ télévision des années soixante, en France, est une télévision d'État, obéissant à une mission de service public. Si le contrôle de l'information constitue un jeu de tensions et de conflits récurrents, les acteurs - journalistes et réalisateurs - partagent néanmoins avec les responsables de programmes une même conviction : la télévision est un outil d'émancipation culturelle des citoyens. Ils adhèrent ainsi à un modèle de télévision s'édifiant sur un projet culturel à dimension pédagogique et à finalité démocratique. ${ }^{(4)} \mathrm{C}^{\prime}$ est sur de telles orientations politiques et culturelles que la télévision se fonde sous la Ivème République ${ }^{(5)}$ orientations confirmées durant les années soixante, quoique soumises non sans heurts, à partir de 1964, à l'épreuve de la croissance. Car les années soixante sont bien celles de l'essor. $13 \%$ des ménages sont équipés d'un récepteur en 1960 et la première et unique chaîne peut être reçue par 50 \% des Français. En 1968, le taux d'équipement des ménages atteint $62 \%$. Le nombre de postes recensés passe ainsi de 1.901 .946 en 1960 à 10.967 .913 en $1970 .{ }^{(6)}$ La réception de la deuxième chaîne, bien qu'encore restreinte en cette période (elle a été créée en avril 1964), vient confirmer le phénomène d'expansion. Nouvelle pratique de loisirs et source d'information des Français, la télévision, désormais installée à l'intérieur de l'espace domestique, prend la famille comme destinataire privilégié. Elle est l'instance à laquelle elle s'adresse. C’est dans ce contexte que se développe, entre 1960 et 1968, les émissions consacrées aux 15-20 ans.

La première émission de télévision qui prend la jeunesse irrégulière pour sujet est une fiction. Elle a pour titre Délinquance juvénile. ${ }^{(7)}$ Réalisée en 1957 par Marcel Bluwal, pour partie en direct, et construite comme 
une pièce de théâtre, elle met en scène un jeune homme parisien de famille aisée, un lycéen confronté au conflit qui l'oppose à son père. Ce jeune homme de la fin des années cinquante va progressivement déserter l'institution scolaire, être accusé du vol du portefeuille d'un camarade, fait qui se révèle exact quand le père découvre que cet argent volé a servi à l'achat d'un scooter. L'histoire s'achève par une fugue et un drame : le jeune homme sera, dans sa fuite, stoppé par un accident. Le drame est alors à son comble quand les parents apprennent qu'une jeune fille accompagnant leur fils est également accidentée. Cette fiction est à la fois une anticipation et une préfiguration d'une thématique ultérieurement développée, celle des conflits générationnels. Elle introduit un questionnement sur les conduites amoureuses des adolescents, ici représentées comme un interdit familial. Cette émission constitue donc un prologue qui ouvre cette phase marquée par l'intérêt grandissant que la télévision française porte à la jeunesse, alors même que « la prise de conscience de l'existence d'une identité propre à la jeunesse est l'occasion, souligne Antoine de Baecque, d'une série d'enquêtes, nombreuses, détaillées, approfondies, au point que l'on peut parler d'un véritable genre possédant ses spécialistes : journalistes, universitaires ou littéraires. Entre 1957 et 1960, journaux, revues, livres, les accueillent à foison. Le mouvement est lancé en mars 1955 par la revue La Nef, qui publie un numéro spécial intitulé "Jeunesse, qui es-tu ?". Puis, en cinq ans, près d'une trentaine d'enquêtes nationales se succèdent $»{ }^{\left({ }^{(8)}\right.}$

Cette fiction, Délinquance juvénile, que la télévision française diffuse en 1957, s'inscrit ainsi à la fois dans cet ample courant d'interrogations sur la jeunesse, tout en annonçant ses propres prises en considération du sujet. Tout d'abord objet de débats en 1958, aux premiers pas de la Ve République naissante, la jeunesse s'installe durablement dans les programmes de télévision à partir de $1960 .{ }^{(9)}$ C'est à la lecture du livre d'Albert Sauvy, La montée des jeunes, ${ }^{(10)}$ que Françoise Dumayet propose et produit le premier magazine pour la jeunesse. D'abord nommé Le journal des jeunes, il prend pour titre définitif L'avenir est à vous. ${ }^{(11)}$ D'autres magazines viendront le compléter, l'étayer comme Le monde en quarante minutes, de Jean-Pierre Gallo et de Jean-Claude Bringuier, en
Moussy et Marcel

Bluwal, réalisation

Marcel Bluwal, première

chaîne, 10 octobre 1957.

(8) Antoine de

Baecque, La nouvelle vague. Portrait d'une

jeunesse, Paris, Flammarion, 1998, p. 51.

(9) Dans la série de débats politiques, $L a$ liberté de l'esprit, animée par Pierre Corval, deux émissions intitulées

"Que veut la jeunesse d'aujourd'hui ?" sont programmées les 14 et 20 novembre 1958.

La première émission est organisée autour du film de Marcel Carné, Les tricheurs ; la deuxième porte notamment sur l'Algérie, mais aussi sur les croyances et valeurs des jeunes présents sur le plateau, confrontés notamment aux questions de Maurice Clavel, Henri Perruchot, auteur de $L a$ France et sa jeunesse... 
Une troisième émission dans la même série,

"Interview sur enfants délinquants”, est programmée le 11 juin 1959.

(10) Alfred Sauvy, La montée des jeunes, Paris, Calmann-Lévy, 1959.

(11) Le journal des jeunes, première chaîne, 18 février $1960-13$ octobre 1960 (21 numéros) ; L'avenir est à vous, de Françoise Dumayet et Jean-Pierre Chartier, première chaîne, 22 octobre 1960-4 mai 1968 (180 numéros).

(12) Le monde en quarante minutes, de JeanPierre Gallo et JeanClaude Bringuier, première chaîne, 10 octobre 1963-7 décembre 1968 (15 numéros sur 42 sont consacrés à la jeunesse).

(13) Seize millions de jeunes, d'André Harris et Alain de Sédouy, deuxiè- me chaîne, 18 avril

1964-8 mai 1968 (117

numéros), et Bouton rouge, deuxième chaîne, 16 avril 1967-11 mai 1968 (26 numéros).

(14) Des métiers et des hommes, première chaîne, 1961-1963 ; La main dans la main, première chaîne, 1964-1966 ; Que ferez-vous demain?, 1964-1967 ; La vocation d'un homme, 1964-1967 ; Jeunesse active, 19661968 ; Demain commence aujourd'bui, 1968-1969.

(15) Anne Pecquet,

L'image des jeunes dans les magazines télévisés (19591970), Mémoire de DEA sous la direction de JeanNoël Jeanneney et Monique Sauvage, Institut d'études politiques, Paris, 1980.

(16) Cinq colonnes à la Une, de Pierre Desgraupes, Pierre Dumayet, Pierre Lazareff et Igor Barrère, première chaîne, octobre 1963, ${ }^{(12)}$ ou encore Seize millions de jeunes et Bouton rouge, d'André Harris et Alain de Sédouy. ${ }^{(13)}$ Cette floraison de magazines documentaires est diffusée selon des rythmes distincts (bi-mensuel, mensuel ou hebdomadaire). Dans ce mouvement régulier de rotations et de relais, s'imprime, dans les grilles de programmes, une permanence du sujet. Outre ces magazines spécifiques, la télévision propose un ensemble d'émissions consacrées à l'apprentissage professionnel et aux métiers. ${ }^{(14)}$ En dernier lieu, il convient de noter que la jeunesse est également une source de sujets des magazines d'information, programmés pour tout public en début de soirée. ${ }^{(15)}$ Elle représente $7 \%$ des sujets de Cinq colonnes à la Une, 8 \% d'entre eux dans le cas de Zoom, $15 \%$ de ceux du magazine d'actualités Panorama. ${ }^{(16)}$ À partir de l'étude de ces documents et de leur contexte de diffusion, se distinguent les contributions de la télévision à la question récurrente, en cette période, de la jeunesse.

\section{La construction de figures antithétiques (1958-1964)}

Plusieurs thèmes traversent L'avenir est à vous, le magazine de Françoise Dumayet : les loisirs et les pratiques culturelles de la jeunesse, l'encadrement de la jeunesse par les associations et leurs différents mouvements. La curiosité et l'initiative sont ainsi des qualités promues, de même que le partage d'expériences au sein de communautés propices à l'exercice des responsabilités, à l'entraide et au respect d'autrui. ${ }^{(17)} \mathrm{Ce}$ pendant, une part importante des émissions, durant cette courte phase, manifeste un intérêt majeur à l'égard du choix et de l'apprentissage d'un métier. Ce 
dernier permet, en effet, l'accès à la vie sociale. Le travail est, ici, présenté comme l'expression de la dignité de l'homme : celle du citoyen. Domaine masculin, le travail est le noyau central, du côté des hommes, de l'édification de leur avenir. L'entrée dans la vie professionnelle, dans L'avenir est à vous, est donc posée comme l'une des étapes constitutives de l'entrée dans l'âge adulte. Elle permet aux jeunes gens - à leur retour du service militaire - de prétendre au mariage, cet acte fondateur de la famille dont ils seront le chef. Le mariage marque alors le changement de statut. Si ces valeurs sont communes aux deux jeunesses que distingue L'avenir est à vous - jeunesse populaire et jeunesse bourgeoise -, elles s'inscrivent selon des trajectoires, des processus d'intégration et des rythmes distincts pour l'une et pour l'autre. Ce modèle de deux jeunesses analysé par Antoine Prost, ${ }^{(18)}$ présent dans la France de l'entre-deux-guerres, subsiste dans ce corps d'émissions hebdomadaires que diffuse la télévision française, un modèle néanmoins infléchi par la présence exemplaire de jeunes de milieux modestes devenus étudiants boursiers et méritants au sein de l'université. À cette division de la jeunesse - patente à l'image dans la mesure où les jeunes apprenti(e)s, ouvrier(e)s ne côtoient pas dans ces émissions les jeunes étudiant(e)s -, vient se superposer un clivage garçonsfilles. Les jeunes filles de L'avenir est à vous, filmées à l'usine, au bureau, en atelier, salariées ou étudiantes, font du mariage la clé de voûte de l'accès à leur vie d'adulte. Le travail s'en trouve renvoyé à la seconde place, inconciliable avec la vie maritale et domestique. Seule l'éventualité du travail à mi-temps est envisagée, et comme solution raisonnable et comme gage suffisant d'indépendance. Dans ce magazine, où les filles et les garçons sont interrogés séparément, s'instaure une césure entre les tâches, les espaces et les rôles. L'avenir est à vous trace des lignes de conduite au sein desquelles s'esquisse la construction sociale d'une jeunesse entreprenante et sage, laborieuse et responsable.

En contrepoint, les écarts aux modalités de passage d'un âge à un autre et aux comportements édictés comme normaux renforcent ce qui relève de la transgression, du désordre, de l'exclusion. Dans cet environnement moral, prennent place les très rares émissions sur la jeunesse irrégulière, c'est-à-dire sur les mineurs ayant eu, pour délits, à janvier 1959-mai 1968.

Sur 556 sujets, 35 por-

tent sur la jeunesse.

Zoom, d'André Harris et Alain de Sédouy, deuxième chaîne, décembre 1965-mai 1968.

Sur 111 sujets, 8 sont consacrés aux jeunes.

Panorama, de Claude Désiré et Gilbert Larriaga, avril 1965-juin 1970, première chaîne (à partir du 1er septembre 1969, Olivier Todd devient responsable du magazine). Sur 180 numéros, 28 portent sur la jeunesse.

(17) Marie-Françoise Lévy, "Les représentations sociales de la jeunesse à la télévision française. Les années soixante", Hermès, n 13 14, 1994, p. 205-217.

(18) Antoine Prost, "Jeunesse et société dans la France de l'entredeux-guerres”, Vingtième Siècle, janvier-mars 1987, p. 35-43. 
(19) Outre La liberté de

l'esprit, "Interview sur enfants délinquants", du 11 juin 1959, six documents - hors journal télévisé - ont été relevés à partir de l'inventaire réalisé (base de données de l'InA, fichiers thématiques, dépouillement des titres et sujets des magazines pour la jeunesse et des magazines d'actualités). Il s'agit de :

- La justice des hommes, "Enfants inadaptés", d'Etienne Lalou et Igor Barrère, première chaîne, 16 novembre 1959 ;

- La justice des hommes, "La délinquance féminine”, d’Étienne Lalou et Igor Barrère, première chaîne, 29 janvier 1960 ;

- Cinq colonnes à la Une, "Square des

Batignolles. Blousons noirs”, première chaîne, 4 novembre 1960 ;

- Cinq colonnes à la Une, "Quarante mille voisins", première chaîne, 2 décembre 1960 ;
- L'avenir est à vous,

"Les jeunes du square

Saint Lambert", de

Françoise Dumayet et Jean-Pierre Chartier,

12 décembre 1960 ;

- Cinq colonnes à la

Une, "Éducation sur-

veillée. La métamorpho-

se”, première chaîne,

6 avril 1963.

Quant au journal télévisé, il consacre, sur cette période, six sujets aux "blousons noirs"

(JT du 23 octobre 1960,

7 février 1961,

16 octobre 1961 ,

21 février 1962,

3 octobre 1963, 6 juillet

1963) ; deux sujets

portent sur les centres d'accueil (JT du 9 septembre et du 15 octobre 1963) ; une "Enquête sur la délinquance juvénile” est diffusée en deux parties (JT des 17 et 31 octobre 1960) et huit sujets sur "la délinquance juvénile” sont diffusés, dont cinq au cours de l'année 1963 (JT du 7 et répondre de leurs actes devant la police ou la justice. ${ }^{(19)}$ L'une des caractéristiques de ces documents réside dans la prudence dont fait preuve la télévision lorsqu'elle s'empare de ce sujet. Le commentaire introductif d'une des premières enquêtes de terrain, "Le square des Batignolles", est ainsi conçu comme un avertissement : "Pilule est le chef de la bande des blousons noirs des Batignolles et Patrice de $C$. est élève du Lycée Carnot qui a ouvert sa cave personnelle, non pour y boire mais pour s'y distraire, aux blousons noirs des Batignolles en même temps qu'à ses camarades de lycée. [...] Dans cette cave donc, précise le commentaire, deux jeunesses se retrouvent. Ce point attire l'attention. Il veut dire qu'une bande de blousons noirs a décidé de sortir de son isolement. [...] À cause de cette attitude nouvelle, nous sommes allés voir cette bande de garçons dont un quart, environ, se trouve en liberté surveillée. Leur lieu de rassemblement est ce café : la Cigale." (20) L'intention, dans ce reportage de Cinq colonnes à la Une, diffusé en novembre 1960, est explicite. Il s'agit certes d'enquêter sur une "bande de jeunes" faisant l'objet d'une intervention éducative en milieu ouvert, mais seulement parce qu'elle fait preuve de sa réinsertion dans une vie régulière et dans la communauté élargie des jeunes. Dans ces conditions, la télévision peut jouer son rôle : informer et donner à réfléchir. Pour conclure son préambule directement adressé aux téléspectateurs, le journaliste de Cinq colonnes peut ainsi préciser son approche et sa démarche à l'égard des jeunes filmés et interrogés : "Les réponses aux questions que je leur ai posées sont souvent brutales, choquantes et presque toujours exprimées dans un langage violent. On ne connaît pas un mal en l'ignorant. Nous avons essayé de savoir, à 
défaut de comprendre, à quelle mentalité correspond le comportement habituel du blouson noir et dans quelle mesure cette mentalité peut ou non évoluer. Au reportage que vous allez entendre et voir nous n'apporterons aucune conclusion laissant à chacun le soin de juger." (21)

Dans ce document, le décor est aussitôt planté : de nuit, des jeunes gens à scooter roulent à vive allure dans les rues de Paris. "Une coloration spécifique de violence" recouvre ces premières images. ${ }^{(22)}$ De ce point de vue, celles-ci collent aux descriptions par lesquelles commencent la plupart des textes sur les blousons noirs et s'ouvrent les films américains aujourd'hui emblématiques (L'équipée sauvage, de Lazlo Benedek, 1953 ; La fureur de vivre, de Nicholas Ray, 1955). À cette atmosphère d'ivresse et de vitesse, de conquête avide d'espace et de territoires, d'étrangeté et d'inquiétude, succède, dans ce reportage télévisé, l'entretien. Il est mené au café avec le groupe de jeunes gens dont le chef est le porte-parole. En premier lieu, un tour d'horizon permet de situer chacun : l'âge, la situation professionnelle, la forme des liens avec la famille, la nature des délits. La présentation des personnages insiste sur les signes distinctifs par lesquels ces jeunes se reconnaissent et s'affirment (les vêtements, la coiffure) et souligne les comportements et les pratiques qui les rassemblent. Dans cette perspective, sont évoquées les provocations qui peuvent aller jusqu'au délit (bruit, tapage nocturne, bagarre), mais aussi les loisirs partagés sans incident dans des lieux collectifs. Ainsi alterne un ensemble multiforme d'attitudes et de points de vue de ces jeunes, oscillant selon une logique d'exclusion-inclusion, elle-même prise dans un mouvement de tensions où se noue le parcours fragile d'une intégration.

Il est intéressant de noter que la construction de ce reportage, en s'inscrivant dans cette dynamique, permet de montrer l'ambivalence des conduites liée, d'une part, aux histoires de ces jeunes souvent en situation de rupture familiale et, d'autre part, à leur révolte ici clairement exprimée envers les formes d'organisation sociale perçues comme une injustice. L'avenir est synonyme, pour eux, de non avenir, d'impasse. La raison majeure qui anime la révolte de ces jeunes de milieu populaire et qui fonde les manifestations de leur opposition réside dans la conscience qu'ils ont d'appartenir à une jeunesse laissée pour compte au
21 novembre 1960, JT du 28 novembre 1961, JT du 13 février 1963, 14 mai et 6 juin 1963, JT du 22 août et 9 décembre 1963).

(20) Cinq colonnes à la Une, "Square des Batignolles. Blousons noirs”, première chaîne, 4 novembre 1960 .

(21) Ibid.

(22) Concernant

l'étude des textes parus, entre 1957 et 1965 , sur les blousons noirs, voir Françoise Tétard, "Le phénomène blousons noirs en France (fin des années cinquante-début des années soixante)", in Révolte et société, Actes du IVème colloque d'Histoire au Présent, Paris, Publications de la Sorbonne, 1989, p. 205-214. 
(23) Cinq colonnes à la Une, "Square des Batignolles. Blousons noirs”, première chaîne, 4 novembre 1960

\section{(24) À cette figure}

féminine du bien vient s'opposer l'image de la jeune fille perdue et déchue, séduite et abandonnée. Ce thème de "la jeune fille en danger" est traité dans les émissions suivantes : Édition spéciale, "Les femmes jouent leur destin", de François Chalais et Frédéric Rossif, première chaîne, 14 juillet 1956 ; Si c'était vous, "Une jeune fille de province", de Marcel Bluwal et Marcel Moussy, première chaîne, 10 juin 1958 ; La justice des hommes, "Délinquance féminine", première chaîne, 29 janvier 1960. regard de ceux qu'ils nomment "les riches", "les vernis", "les snobs”, en un mot, les autres, "les étudiants". Ce sentiment d'inégalité de situation et d'avenir, entre ceux qui travaillent et ceux qui poursuivent leurs études, se trouve renforcé par l'inégalité devant l'appel sous les drapeaux et le départ pour l'Algérie entre les étudiants, qui bénéficient d'un sursis, et eux, bons pour la guerre.

Si donc Françoise Dumayet, dans son magazine L'avenir est à vous, montre, au cours de ces années 1958-1964, l'existence persistante de deux jeunesses (populaire et bourgeoise), avec leurs propres rites de passage et d'intégration, les témoignages de jeunes diffusés dans le reportage de Cinq colonnes à la Une introduisent une remise en cause de cette organisation sociale, définissant et les modalités d'entrée dans la vie adulte et les trajectoires sociales qu'elles sous-tendent. Ce document de Cinq colonnes souligne ainsi les raisons de la colère dans un environnement où les jeunes interrogés font néanmoins preuve de leur cheminement pour rejoindre le parcours social qui leur est tracé. Et dans cette logique d'exclusion-inclusion qui structure ce document télévisé sur la jeunesse irrégulière, se retrouvent trois éléments : passer de la révolte et de la violence à la raison, passer de l'inactivité au travail, cicatriser les blessures d'une enfance heurtée en trouvant des adultes, éducateurs et compagnons de route. Reste l'amour, timide, rédempteur, espéré comme couronnement et comme récompense de cet âpre apprentissage de la jeunesse. Comme le dit un jeune homme du "Square Saint-Lambert" : "En général il n'y a qu'une chose qui peut faire changer un gars... soit une fille, soit les coups. En général les coups ne réussissent pas mais les filles réussissent assez bien." (23) L'évocation de l'amour est ici associée à l'image d'une jeune fille, incarnation du réconfort, de la tendresse, de la douceur, celle-là même qui fait fléchir la violence, panse les blessures, apaise et permet la métamorphose du "mauvais garçon". ${ }^{(24)}$ Et cette évocation de l'amour clôt ce document qui, sans procédé dramatisant mais non sans effets de mise en scène, propose une vision morale - explicative et non stigmatisante - des attitudes d'une jeunesse moins rebelle qu'en révolte et en lisière de rupture sociale, fait résultant d'oppositions et de clivages au cœur d'une même génération, alors que sévit la guerre d’Algérie. 
Paré d'une dimension exemplarisante - ces jeunes gens des quartiers populaires de Paris sont désormais sur la bonne voie et peuvent faire entendre le témoignage à vif de leur expérience - ce document, dans son propos, présente également la caractéristique d'installer visiblement la présence et la représentation de l'instance judiciaire comme force et comme réponse aux actes délictueux commis par des mineurs. Épousant l'esprit de l'ordonnance du 2 février 1945 sur l'enfance délinquante - qui promeut la notion d'éducabilité et fonde la décision du juge plus sur la personnalité du mineur que sur la matérialité des faits - et les principes de l'ordonnance du 23 décembre 1958 sur l'enfance en danger - dont la mission est préventive -, ${ }^{(25)}$ les reportages de Cinq colonnes à la Une, "Le square Saint-Lambert" et "La métamorphose", (26) montrent une justice des mineurs en action, qu'il s'agisse, dans un cas, d'intervention en milieu ouvert et, dans l'autre, du fonctionnement d'une institution (le centre de Savigny-sur-Orge). Ainsi coexistent dans ces documents une approche d'investigation d'un fait social, la délinquance, le rappel des règles et normes d'apprentissage de la jeunesse, la réponse d'ordre juridique aux infractions commises par des mineurs.

Cette construction en triptyque dans des documents dont la rareté est, rappelons-le, frappante au regard de la présence régulière de la jeunesse à l'écran, renforce le caractère exceptionnel de l'intervention de la télévision sur la question de la délinquance. Discrétion qui, associée au caractère solennel que prennent ces quelques intrusions dans l'univers de la jeunesse irrégulière, a une double portée : elle laisse entendre que ces situations d'irrégularité sont circonscrites à quelques groupes - voire à quelques cas - et qu'elles sont maîtrisées par les processus judiciaires auxquels elles donnent lieu. Loin de contribuer à l'amplification du phénomène "blouson noir", la télévision adopte une position de retrait ou d'absence réfléchie pour intervenir de façon circonstanciée sur ce sujet, tout en privilégiant le traitement d'une question sociale, celle de la jeunesse, en s'appuyant sur l'exemple. Ainsi peut-on envisager l'hypothèse selon laquelle les rares enquêtes et documents sur les bandes, sur leurs écarts de conduite, ne sauraient percuter ou troubler l'image pacifique d'une jeunesse vaillante, douée de raison, porteuse des espoirs d'une
(25) Jacques Bourquin, "Chronologie succincte de l'Éducation surveillée", Pour, n ${ }^{\circ} 110 / 111,1987$, p. 101-108.

(26) Cinq colonnes à la Une, "La métamorphose”, première chaîne, 6 avril 1963. 
(27) Dans le magazine

L'avenir est à vous (première chaîne) : "Jeune dans une ville neuve", 10 septembre 1964 ;

"Les jeunes dans la rue", 16 février 1966 ;

"Jeunes gens dans leur

banlieue" ou "Banlieue en extension. Bobigny”, 20 juin 1966 ; "Éducation surveillée. Juge pour enfants", 29 octobre 1966. Dans Seize millions de jeunes (deuxième chaîne) :

"Habitations à loisirs modérés”, 3 décembre 1964 ; "Sont-ils bons, sont-ils méchants ?”, 22 avril 1965 ; "La bande”, 20 septembre 1966 ; "La bécane", 13 décembre 1966.
(28) Dans la série À la découverte des Français, de Jean-Claude Bergeret avec le concours de Paul-Henry Chombart de Lauwe et du groupe d'ethnologie sociale du Musée de l'homme, deux documents traitent de ces questions :

"Rue du Moulin de la Pointe”, réalisation Jacques Krier, première chaîne, 5 avril 1957, et "La Butte à la Reine", réalisation Jean-Claude Bergeret, première chaîne, 12 avril 1957.

(29) Dans l'ouvrage, sous la direction de Paul-Henry Chombart de Lauwe, Famille et habitation, 2 vol., I. Sciences humaines et conception
France qui a retrouvé son unité et sa prospérité. $A$ fortiori, ils contribuent, en ce qui concerne le positionnement de la télévision, à renforcer cette approche consensuelle.

\section{Le malaise de la jeunesse : le tournant de 1964}

Comment ce consensus qui définit l'attitude de la télévision envers la jeunesse et son rôle de régulation des personnes et des familles se trouve-t-il, au tournant de 1964, infléchi, déplacé, fracturé ? Par l'intrusion de nouveaux regards portés par des journalistes dans leurs magazines pour la jeunesse, tels André Harris et Alain de Sédouy dans Seize millions de jeunes. Ils engagent une réflexion corrosive et critique sur les difficultés sociales, matérielles, existentielles d'une jeunesse, non plus organisée à son entrée dans l'âge adulte selon des modalités d'intégration propres au milieu d'appartenance sociale, mais rassemblée par l'âge, les goûts, les loisirs, la musique, les pratiques de consommation, les difficultés financières, un sentiment d'isolement et de coupure avec les adultes, finalement les conflits entre générations. Ainsi, ce magazine contribue-t-il à défaire ce modèle où coexistaient deux jeunesses - de milieux populaires et bourgeoise - et à comprendre la jeunesse comme une classe d'âge, indépendante du parcours social de chacun, unie par une culture, des intérêts communs et des interrogations partagées sur le monde dans lequel elle devra prendre place. Un double renversement s'opère à travers ce nouveau magazine, l'un est propre à une perception de la jeunesse, l'autre concerne le rôle d'une société dès lors jugée potentiellement responsable du malaise latent de ce groupe social lié 
par l'âge, une société laissant vacantes ou inadaptées les réponses face aux questions que ce malaise suscite.

C'est dans ce contexte de mutation que s'inscrivent les émissions toujours peu nombreuses - consacrées à la jeunesse irrégulière. ${ }^{(27)} \mathrm{Ces}$ dernières n'articulent plus leur propos sur une logique d'exclusion et d'inclusion concernant des groupes restreints de jeunes vivant dans l'enceinte de la Capitale, mais elles se fondent sur l'observation d'une marginalité juvénile considérée comme un phénomène diffus : un nouveau fait de société, écho exacerbé du malaise de la jeunesse, qui prend racine dans les grands ensembles de la région parisienne. Certes, les premiers reportages de télévision sur l'expansion des villes à la périphérie de Paris datent de la fin des années cinquante. Ils présentent pour caractéristique d'enquêter sur les impressions de frayeur ou d'écrasement que ce nouvel urbanisme provoque, sur l'isolement de ces lieux par rapport à Paris, sur l'effacement de la vie sociale. Paul-Henry Chombart de Lauwe, dans une série documentaire de Jean-Claude Bergeret et de Jacques Krier, avait, en avril $1957,{ }^{(28)}$ contribué à faire réfléchir les téléspectateurs sur l'indispensable nécessité d'inventer, dans ces nouveaux lieux de résidence, de nouvelles formes de relations de voisinage solidarités et entraide - pour que se reconstituent, à travers des liens entre individus, des groupes sociaux - "des groupes d'habitation" (29) favorisant l'intégration des personnes à une vie collective dynamique. Si Chombart et son équipe mettaient l'accent sur la reconstruction de pratiques sociales pour donner sens à la vie quotidienne des habitants de ces résidences, les reportages des années soixante sur les cités récentes de la banlieue parisienne s'accordent sur l'amélioration des conditions matérielles de vie (logement plus grand, équipement sanitaire satisfaisant), mais distillent le doute sur une vie humaine à venir possible au regard de l'architecture des grands ensembles engendrant anonymat et repli des ménages sur eux-mêmes. ${ }^{(30)}$ L'absence d'équipements sociaux, ainsi que l'architecture et l'immensité des lieux sont mis en avant jusqu'à constituer insensiblement des éléments définissant ces banlieues comme des lieux porteurs d'inconnu et d'étrangeté, des lieux à part, des lieux de vies éparses et suspendues, des lieux pouvant engendrer la délinquance. de l'habitation, 220 p.,

II. Un essai d'observation

expérimentale, 374 p.,

Paris, éditions du CNRS, 1959-1960, « l'habitation dans la vie sociale » comprend « tout ce qui concerne la place qu'occupe l'habitation dans la vie des hommes, et toutes les questions techniques, économiques, culturelles qui s'y rapportent ». " La vie sociale dans l'habitation » inclut « les comportements des hommes et les rapports qui s'établissent entre eux à l'intérieur du logement pour le ménage et à l'intérieur des groupes d'habitation pour des ensembles de ménages " (vol. I, p. 15).

(30) Voir, notamment, Cinq colonnes à la Une, "Quarante mille voisins”, première chaîne, 2 décembre 1960. 
(31) Voir Henri Boyer et Guy Lochard avec la participation d'André Bercoff, Scènes de télévision en banlieues, 19501994, Paris, L'Harmattan/INA, coll. "Mémoires de télévision”, 1998, $201 \mathrm{p}$.

(32) Seize millions de jeunes, d'André Harris et Alain de Sédouy, "Habitations à loisirs modérés”, journaliste Jean-Paul Thomas, deuxième chaîne, 3 décembre 1964.

(33) Ibid.
Ainsi s'édifie imperceptiblement, à l'orée des années soixante, l'idée selon laquelle les hommes vivant dans ces grands ensembles sont autres, marqués par cet urbanisme qui fascine et effraie, ou bien détruit : idée qui trouve son déploiement dans les années soixante-dix. ${ }^{(31)}$

C'est dans ce contexte d'interrogations sur l'habitat neuf et désolé, d'une part, sur la jeunesse rassemblée par des difficultés économiques, matérielles et existentielles, d'autre part, que prennent place les documents télévisés sur la jeunesse non plus irrégulière, mais en voie de marginalisation. Les reportages se situent dans les cités neuves de la périphérie de Paris (La Courneuve, Sarcelles, Romainville...). Ils concernent des jeunes dont certains seulement sont à la lisière de la délinquance. La marginalité, dans ces reportages, résulte donc moins d'actes de délinquance que d'un sentiment d'abandon, de rejet des adultes, de mise à l'écart, sentiment ressenti et partagé par ces jeunes, qui y répondent par une sourde hostilité au monde dans lequel ils vivent. Dans un document privilégiant cette approche, "Habitations à loisirs modérés", ${ }^{(2)}$ les auteurs avancent plusieurs explications : la société marchande, axée sur la promotion de la consommation (disques, revues, électrophones, transistors, vêtements), grossit chez des jeunes disposant de peu de revenus ou d'argent de poche, un sentiment d'exclusion et d'injustice qui peut conduire jusqu'au vol. L'ennui des jeunes pèse, créé par l'absence de structures sportives ou culturelles. "Les jeunes s'ennuient, commente l'un des journalistes de ce reportage. Et le résultat, on les voit, au milieu des blocs, se promener dès qu'ils ont un moment de libre, se promener en mobylette et errer, comme ça, à la grande frayeur de la population." La réaction de l'autre journaliste, en voix off, précise : "Mais ils se promènent comme ça, gratuitement, je veux dire, ils vont d'un endroit à un autre, mais pas forcément à des endroits précis..." ${ }^{(33)}$ Les désaccords et les tensions entre parents et enfants ont pour conséquence de pousser les jeunes hors du domicile familial, dans la rue, où ils attendent que le temps passe. Cheveux longs, attitudes vestimentaires, demandes d'argent ou d'autorisations diverses alimentent les discussions et les conflits entre générations. Dans ce même reportage, on peut entendre : "Par exemple, qu'est-ce que tu as comme idée et que n'aurait pas ton père?" "Quand je lui dis que je voudrais bien passer mon permis de conduire, il me dit 
non. Tu es encore trop jeune, moi, à ton âge, je n'avais pas de voiture, je faisais la guerre..., des trucs comme ça, alors terminé." ${ }^{(34)}$ Enfin, "l'absence d'âme" des grands ensembles, ces villes en chantier qui renforcent l'anonymat et l'isolement, constitue la dernière cause invoquée pour tenter de comprendre ces comportements et les sentiments de marginalité des jeunes.

Les grands ensembles des banlieues seraient donc producteurs de l'errance, du vagabondage, du désœuvrement de la jeunesse. Une jeunesse isolée de l'autre ville, Paris, ses quartiers, ses lumières, ses cafés. Par ailleurs, les conflits parents-enfants viennent, dans ces lieux d'habitation inanimés, renforcer la solitude. Cette approche du malaise de la jeunesse laisse percer une inquiétude diffuse se constituant comme une intrigue. Le document "Habitations à loisirs modérés" s'achève par cet ultime dialogue entre les deux journalistes : "Le problème consisterait, pentêtre, à tenter qu'ils [ces jeunes] ne s'ennuient pas, c'est-à-dire qu'ils ne soient pas ancrés dans l'ennui." "C'est certain. En fait ce n'est peut-être pas un problème de moyens. Mais un probleme de civilisation." Ainsi, cette courte période - les années 1964-1968 - voit-elle poindre un phénomène : celui d'une mise en intrigue des comportements de la jeunesse, laissant entrevoir qu'une rupture s'opère entre les jeunes et leurs milieux de vie, entre les jeunes et leurs familles, entre les jeunes et leur perception de l'avenir. Cette mise en intrigue introduit et diffuse l'incertitude, l'inquiétude, l'incompréhension des adultes : autant d'éléments qui contribuent à construire socialement - et médiatiquement - les symptômes du malaise de la jeunesse et des formes de marginalité juvénile.

\section{Conclusion}

L'étude des documents produits et diffusés par la télévision montre que s'opère, au cours des années soixante, une évolution des conceptions de la jeunesse : on passe d'une "jeunesse irrégulière" à une "jeunesse marginale". L'affirmation d'une position morale et normative construite autour d'un ordre, de ses règles et de ses transgressions approchés sous forme descriptive - cristallisée autour des bandes et de la figure de l'exclu à laquelle font écho la sanction et la loi - fait face au reflux de la représentation de la justice. La mise en visibilité de la justice des mineurs, 
engageant un processus social et psychologique d'insertion des jeunes, s'efface au moment où la jeunesse, au tournant de 1964, est comprise comme un ensemble de personnes liées par l'âge, prises dans un malaise insaisissable et une marginalité résultant de la distance qui les sépare de leur environnement adulte. Il est donc, désormais, non plus question de délinquance touchant des jeunes rassemblés, dans les quartiers de Paris, en bandes identifiées, mais d'un phénomène diffus, d'un fait de société qui sourd dans les grands ensembles de la région parisienne, environnement favorable à l'éclosion d'attitudes exacerbant et renforçant l'expression d'une marginalité juvénile latente.

Si donc la télévision fait preuve sur ces sujets d'une quasi-absence, troublante, il importe d'observer que cette absence résulte de causes distinctes. Jusqu'au milieu des années soixante, il importe de ne pas donner d'ampleur, en les montrant, à des comportements relevant de la délinquance, afin de ne pas briser l'image de l'unité morale de deux jeunesses. À cette approche, succèdent une interrogation, un doute, une inquiétude à l'égard de ce modèle qui se défait, dévoilant une jeunesse dans son mal-être, qui comprend et suscite des manifestations visibles et neuves pouvant ou non donner lieu à des actes délinquants, mais relevant d'un symptôme général. De ce point de vue, l'absence de reportages ou d'enquêtes spécifiques sur la jeunesse irrégulière peut se comprendre dans le déplacement de la perception de la jeunesse. Néanmoins, force est de constater que si des paramètres de ce malaise, l'ennui, l'errance, l'isolement, la solitude, les conflits de générations, sont propres à la jeunesse, ils sont aussi observés attentivement - en quelques occasions - dans un cadre urbain neuf, porteur de ces troubles et concourrant spécifiquement à les aggraver. Ainsi sont palpables des indices de l'instruction médiatique de "la question des banlieues" associée aux formes de marginalité juvénile - prenant corps à la télévision dans les années soixante-dix -, à partir d'un regard porté par Paris sur sa périphérie. 